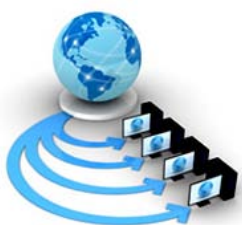

Volume 9, No. 2, March-April 2018

International Journal of Advanced Research in Computer Science

RESEARCH PAPER

Available Online at www.ijarcs.info

\title{
FUZZY THEORY - A SURVEY ON ITS FOUNDATIONS AND ADVANCEMENTS
}

\author{
Saniya Tasleem Zafar \\ M. Tech. Scholar, Department of CSE \\ School of Engg. Sciences and Technology, Jamia Hamdard \\ New Delhi, India
}

\author{
Dr. Safdar Tanweer \\ Assistant Professor, Department of CSE \\ School of Engg. Sciences and Technology, Jamia Hamdard \\ New Delhi, India
}

\author{
Nafisur Rahman \\ Assistant Professor, Department of CSE \\ School of Engg. Sciences and Technology, Jamia Hamdard \\ New Delhi, India
}

\begin{abstract}
The term 'Fuzzy' means vague, unclear, or imprecise. Fuzzy Logic is a many-valued logic that is based on the theory of Fuzzy Sets and it facilitates the representation of approximate reasoning. It finds its use in various areas where binary representations do not suffice. In this paper, we have confined our discussions on the theoretical foundations and advancements of modern Fuzzy Logic. We start with a brief account of Fuzzy Sets followed by the operations they support. Then we discuss how the Linguistic Variables allow more realistic reasoning as opposed to traditional binary reasoning. Then we introduce the theoretical aspects of the calculus of Fuzzy Restrictions. Finally, we discuss the theory of possibility as an alternative to the theory of probability. For the sake of simplicity and intelligibility, we have tried to avoid incommodious mathematical equations throughout this paper.
\end{abstract}

Keywords: Fuzzy Sets; Approximate reasoning; Linguistic Variables; Fuzzy Restriction; Possibility theory

\section{INTRODUCTION}

The theoretical foundations of Fuzzy Logic are based on Fuzzy Sets that deal with the information that is uncertain, generic, imprecise, or vague. The theory of Fuzzy Sets is associated with the idea of membership function by the course of human thinking and understanding. It also gives an effective means for understanding better assessment options. Fuzzy Sets are being used extensively in the development of intelligent systems. Fuzzy Sets are distinguished from crisp sets in that they cater to the requirements of approximate reasoning. Very often, Linguistic Variables are used for this purpose. A Linguistic Variable is a value which is given in the form of word or sentences rather than in a numeric form. Fuzzy Restrictions allow the expression of Fuzzy propositions in the form of relational assignment equations. Treating the incomplete information as a vague concept, the theory of Possibility provides an alternative to Probability theory by dealing with certain types of ambiguity.

\section{FUZZY SETS}

Before discussing Fuzzy Sets [1, 2], it is imperative to define Fuzzy Logic. Fuzzy Logic is a contrast of Boolean logic. Boolean logic consists of value either true or false on the other hand, Fuzzy Logic consists of all the values which come in between completely true to completely false. The fundamental difference between Fuzzy Logic and Boolean logic is that it gives a sliding measure rather than a discrete value. Any value between 0 and 1 , it may be $0.45,0.8,0.978$, and so on and could be represented in a Fuzzy system whereas, the Boolean system will only accept the value 0 or 1 , or true or false.

Zadeh introduced Fuzzy Sets in his seminal paper on the subject in 1965. Fuzzy Sets contain elements with have different orders of membership. They are characterized by a membership function which is valued in the interval $[0,1]$.
Fuzzy Set is, in essence, a class of objects with a continuum of grades of membership.

While Fuzzy Set is an augmentation of the crisp set - a set in which the elements are present or not present. A crisp set is often described as an ordinary or classical set.

The membership function is defined as the mapping of each point in the input value to a degree of membership or membership value which is marked in between the values of 0 and 1.

\section{FUZZY SET OPERATIONS}

The operations on Fuzzy Set are a generalization of the crisp set. The most extensively used operations are Intersection, Union, and Complement [2, 3].

Let $\mathrm{M}$ and $\mathrm{N}$ be the two Fuzzy Sets where both belong to the universal set $\mathrm{U}$.

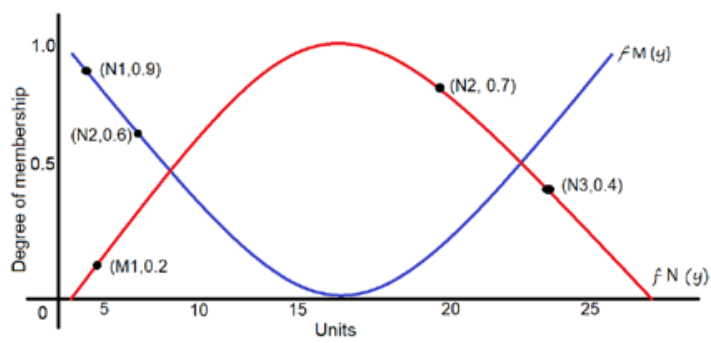

Fig. 1

Intersection Operation: The intersection between two Fuzzy Sets $\mathrm{M}$ and $\mathrm{N}$ is determined by the degree of membership using the function operation:

$$
\mu(\mathrm{M} \cap \mathrm{N})(\mathrm{y})=\max [\mu \mathrm{M}(\mathrm{y}) \cap \mu \mathrm{N}(\mathrm{y})], \mathrm{y} \in \mathrm{U}
$$




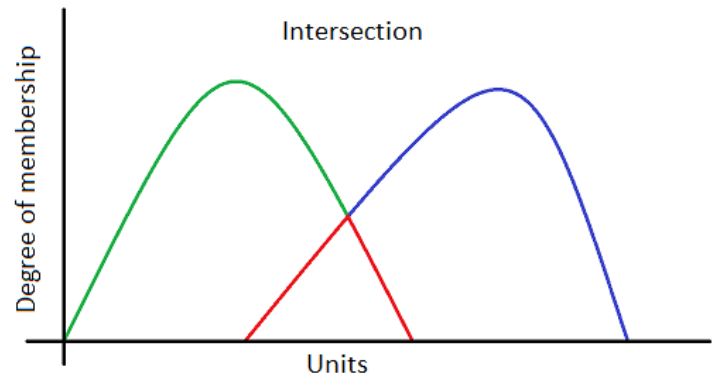

Fig. 2

Union Operation: The union between two Fuzzy Sets M and $\mathrm{N}$ is determined by the degree of membership using the function operation:

$$
\mu(\mathrm{M} \cup \mathrm{N})(\mathrm{y})=\max [\mu \mathrm{M}(\mathrm{y}) \cup \mu \mathrm{N}(\mathrm{y})], \mathrm{y} \in \mathrm{U}
$$

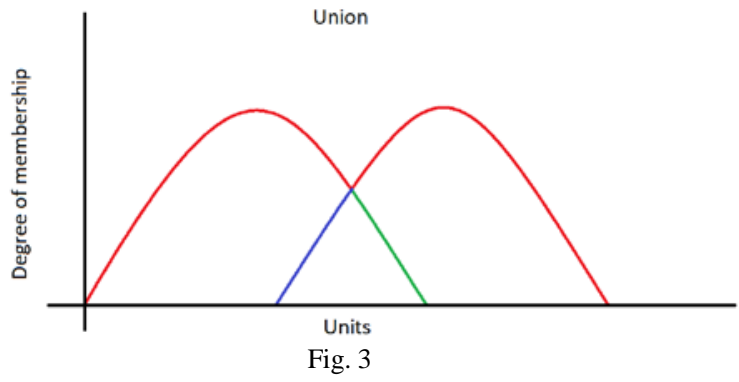

Complement Operation: The complement between two Fuzzy Sets $\mathrm{M}$ and $\mathrm{N}$ is determined by the degree of membership using the function operation:

$$
\mu \tilde{\mathrm{A}}(\mathrm{y})=1-\mu \mathrm{M}(\mathrm{y}), \mathrm{y} \in \mathrm{U}
$$

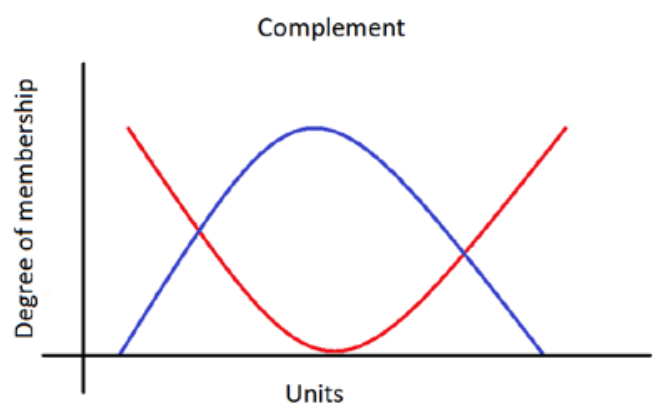

Fig. 4

The following laws are commonly used in classical set theory as well as have been applied in Fuzzy Set theory:

Associative Law:

$(\mathrm{M} \cup \mathrm{N}) \cup \mathrm{P}=\mathrm{M} \cup(\mathrm{N} \cup \mathrm{P})$

$(\mathrm{M} \cap \mathrm{N}) \cap \mathrm{P}=\mathrm{M} \cap(\mathrm{N} \cap \mathrm{P})$

Commutative Law:

$\mathrm{M} \cup \mathrm{N}=\mathrm{N} \cup \mathrm{M}$

$\mathrm{M} \cap \mathrm{N}=\mathrm{N} \cap \mathrm{M}$

Distributive Law:
De Morgan’s Law:

$(\mathrm{M} \cup \mathrm{N})^{\prime}=\mathrm{M}^{\prime} \cap \mathrm{N}^{\prime}$

$(M \cap N)^{\prime}=M^{\prime} \cup N^{\prime}$

\section{LINGUISTIC VARIABLES IN FUZZY SETS}

In 1975, Zadeh introduced the concept of a Linguistic Variable [4, 5] for approximate reasoning. A Linguistic Variable is a variable represented by words or sentences in a human or artificial language. A Linguistic Variable represents crisp information in a pattern and preciseness relevant for the problem. For instance, size is a Linguistic Variable if its values are linguistic rather than numerical i.e. short, tall, very tall, not short, not very tall, etc., instead of $1,2,3,4, \ldots$

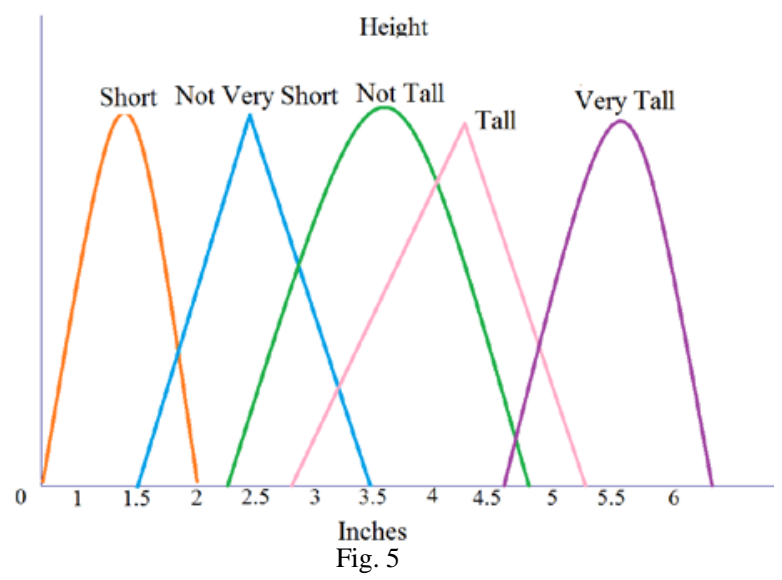

A Linguistic Variable is used in day to day in our life to signify what is vital in its context. For instance, consider the preparation instruction of tea making. These instructions are filled with different linguistic references as in the following: "Bring to boil", "Slow down the flame”, "Partially cover", etc. They use Linguistic Variables within the context of tea preparation. Linguistic Variables allow approximate description and representation where the complexity or definition doesn't allow a precise description that is appropriate. For example, "Falsehood" can be treated as a Linguistic Variable with values such as False, Partially False, Completely False, Not False, etc. This allows the reasoning in Fuzzy Logic. It is more realistic than conventional binary logic as it provides a basis for approximate reasoning, where exactness is not the main concern. Probabilities can also be represented as with values such as highly probable, moderately probable, highly improbable, etc. Such computations require complex non-linear solutions. A Linguistic Variable is combined with the logical Boolean expressions, for instance, Linguistic Variables can be combined with and, or, and not operators. Fuzzy 'And' is the smallest degree of membership of its argument. Fuzzy 'Or' is the highest degree of membership of its argument and Fuzzy 'Not' is the space between the argument and Fuzzy 1. If the values 0 and 1 is reduced during the resolution, then the logical definition for manipulating Linguistic Variable will stay same as conventional Boolean logic. The major applications of the Linguistic Variables are in the areas of intelligent systems, Natural Language Processing, decision processes, clinical diagnostics, information retrieval, pattern recognition and various allied areas.

$M \cup(N \cap P)=(M \cup N) \cap(M \cup P)$
$M \cap(N \cup P)=(M \cap N) \cup(M \cap P)$ 


\section{FUZZY RESTRICTIONS}

In 1975, Zadeh came up with the calculus of Fuzzy Restrictions [6] that can be viewed as a branch of the Fuzzy Relations' theory. It is concerned mainly with the translation of proposition of variety of type into relational assignment equation. In general, a composite Fuzzy proposition [7] translates into a system of relational assignment equations. The Fuzzy Restrictions may be modified by employing the concepts of concentration and dilation. The calculus of Fuzzy Restrictions applies to approximate reasoning where exactness is not the primary concern. Compatibility functions and compatibility matrices for base variables are used to simplify the tasks where there is no requirement for high-level preciseness.

\section{THEORY OF POSSIBILITY}

Zadeh introduced the theory of possibility [8] in 1978 as an addition to Fuzzy Sets and Fuzzy Logic theory. Possibility theory treats incomplete information as a vague concept. It is an alternative to probability theory. The link between these two theories is based on set functions. Both the theories deal with uncertainty and measure in the range of $[0,1]$ interval. Possibility theory has enabled a typology of a rule to be exposed, categorizing rules whose purpose is to propagate random error through reasoning steps. Most of the application of Fuzzy Sets theory relates to the concept of a Fuzzy Restriction. It acts as an elastic constraint on the values that are assigned to a variable. In terms of restriction, the essence of a proposition of the form "Y is S", where $\mathrm{Y}$ is the name of an object and $\mathrm{S}$ is the Fuzzy Set, may be expressed as a relational assignment equation of the form $\mathrm{R}(\mathrm{B}(\mathrm{Y}))=\mathrm{S}$, where $\mathrm{B}(\mathrm{Y})$ is an implied attribute of $\mathrm{Y}, \mathrm{R}$ is a Fuzzy Restriction on $\mathrm{Y}$ and $\mathrm{S}$ is the unary Fuzzy relation which is assigned to R. For example: "Jenny is fat", where fat is a Fuzzy subset of the real line, translates into $\mathrm{R}($ Size(Jenny)) = Fat. Fuzzy Logic is based on the observation that human perception involves, for the most part, Fuzzy Sets, that is classes of objects in which conversion from members to nonmembers is steady rather than abrupt. It is emphasizing the proposition line 'Jay is very smart', 'Maya is taller than John', 'Most serials are not thrilling', etc. we shall refer to such declarations as Fuzzy propositions in order to comprehend them from non-Fuzzy propositions like "All women are mortal", " $\mathrm{n}$ is smaller than m", etc.

\section{ConClusions}

In the preceding discussions, we have presented the founding principles of Fuzzy theory and its subsequent advancements for which Prof. Zadeh was almost singlehandedly responsible. The works in this area are still fascinating the researchers and, in all likelihood, will continue to do so in future.

\section{REFERENCES}

[1] Zadeh, L.A. "Fuzzy sets”. Information and control, vol. 8, no. 3, pp. 338-353, 1965.

[2] Bellman R., Kalaba R., and Zadeh L.A. “Abstraction and pattern classification”. Journal of Mathematical Analysis and Applications, vol. 13, no. 1, pp. 1-7, 1966.

[3] Halmos, P.R. Naive Set Theory. New York: Van Nostrand, 1960.

[4] Zadeh, L.A. "The concept of a linguistic variable and its application to approximate reasoning-I”. Information sciences, vol. 8, no. 3, pp. 199-249, 1975.

[5] Zadeh, L.A. "The concept of a linguistic variable and its application to approximate reasoning-II”. Information sciences, vol. 8, no. 4, pp. 301-357, 1975.

[6] Zadeh L.A. “Calculus of Fuzzy Restrictions,” in Proc. US-Japan Seminar on Fuzzy Sets and their Applications, 1975, pp. 1-39.

[7] R. E. Bellman and L. A. Zadeh. "Local and fuzzy logics" in Modern Uses of Multiple-Valued Logics, edited by J. M. Dunn and G. Epstein, D. Reidel, Dordrecht-Holland, 1977, pp. 103 165.

[8] Zadeh, L.A. "Fuzzy sets as a basis for a theory of possibility”. Fuzzy Sets and Systems, vol. 1, no. 1, pp. 3-28, 1978. 